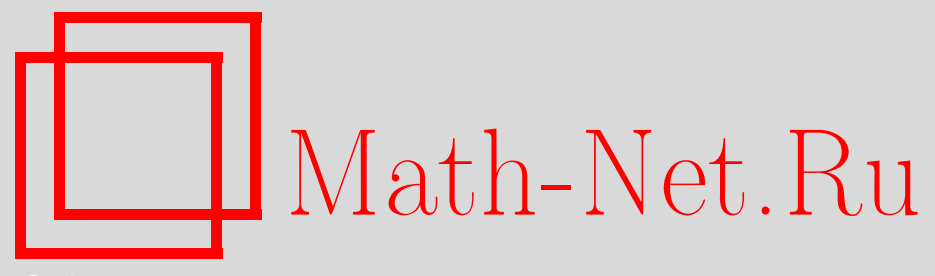

С. М. Заикина, Обобщённое интегральное преобразование Лапласа и его применение к решению некоторых интегральных уравнений, Вестн. Сам. гос. техн. ун-та. Сер. Физ.-мат. науки, 2014, выпуск 1(), 19-24

DOI: https://doi.org/10.14498/vsgtu1265

Использование Общероссийского математического портала MathNet.Ru подразумевает, что вы прочитали и согласны с пользовательским соглашением

http: //www . mathnet.ru/rus/agreement

Параметры загрузки:

IP: 54.198 .64 .247

26 апреля 2023 г., 12:43:03

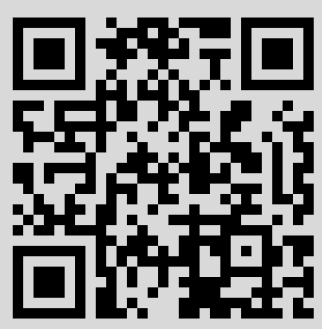


УДК 517.442+517.581

\title{
ОБОБЩЁННОЕ ИНТЕГРАЛЬНОЕ ПРЕОБРАЗОВАНИЕ ЛАПЛАСА И ЕГО ПРИМЕНЕНИЕ К РЕШЕНИЮ НЕКОТОРЫХ ИНТЕГРАЛЬНЫХ УРАВНЕНИЙ
}

\author{
C. М. Заикина ${ }^{1,2}$ \\ 1 Самарский государственный технический университет, \\ Россия, 443100, Самара, ул. Молодогвардейская, 244. \\ 2 Волгоградский государственный университет, \\ Россия, 400062, Волгоград, пр. Университетский, 100.
}

Рассматриваются обобщённые интегральные преобразования Лапласа, которые в ядре содержат обобщённую конфлюэнтную гипергеометрическую функиию ${ }_{1} \Phi_{1}^{\tau, \beta}(a ; c ; z)$. C использованием свойств этих преобразований для них получен аналог теоремы о свёртке. Методом интегральных преобразований решены интегральные уравнения Вольтерра первого рода, содержащие в ядре конфлюэнтную гипергеометрическую функиию. При решении интегральных уравнений использовались формулы обращения введённых интегралъных преобразований, полученные автором ранее.

Ключевые слова: интегральное преобразование Лапласа, интегральные уравнения, обобщённая гипергеометрическая функиия.

Метод интегральных преобразований широко используется для решения дифференциальных и интегральных уравнений, а сама теория интегральных преобразований является одной из важных ветвей прикладного анализа. Многие задачи математической физики, астрофизики, термодинамики, механики и других естественных наук приводят к необходимости применения теории интегральных преобразований.

Преимущества метода интегральных преобразований заключаются в том, что он даёт возможность:

1) сведения сложных задач к менее сложным;

2) получения окончательного результата в явном виде.

Разработкой теории и методов интегральных преобразований занимались Г. Бейтмен, В. А. Диткин, А. А. Килбас, А. П. Прудников, С. М. Ситник, А. Эрдейи, М. Saigo, I. N. Sneddon [1-4] и многие другие.

Изучение интегральных уравнений первого рода и так называемых парных, тройных и $N$-арных интегральных уравнений, которые часто встречаются в приложениях, приводит к необходимости рассматривать интегральные

ISSN: 2310-7081 (online), 1991-8615 (print); doi: http://dx.doi.org/10.14498/vsgtu1265 (C) 2014 Самарский государственный технический университет.

Образец цитирования: С. М. З аи кина, "Обобщённое интегральное преобразование Лапласа и его применение к решению некоторых интегральных уравнений" // Becmн. Сам. гос. техн. ун-та. Сер. Физ.-мат. науки, 2014. № 1 (34). С. 19-24. doi: 10.14498/vsgtu1265.

Сведения об авторе: Светлана Михайловна Заикина, аспирант, каф. прикладной математики и информатики ${ }^{1}$; ассистент, каф. компьютерных наук и экспериментальной математики ${ }^{2}$.

E-mail address: svetzai@inbox.ru 
преобразования со специальными функциями в ядрах. Эти вопросы изучены в работах $[3,5]$.

Рассмотрим обобщённые интегральные преобразования Лапласа:

$$
\begin{gathered}
\widetilde{\mathcal{L}}\{f(t) ; x\}=\int_{0}^{\infty} \exp (-t x)_{1} \Phi_{1}^{\tau, \beta}\left(a ; c ;-b(t x)^{\gamma}\right) f(t) d t, \\
\widetilde{\mathcal{L}}_{\gamma_{1}, \gamma_{2}, \gamma}\{f(t) ; x\}=\int_{0}^{\infty} t^{\gamma_{2}} \exp \left(-(x t)^{\gamma_{1}}\right)_{1} \Phi_{1}^{\tau, \beta}\left(a ; c ;-b(t x)^{\gamma \gamma_{1}}\right) f(t) d t,
\end{gathered}
$$

где $t \geqslant 0 ; \gamma \in \mathbb{C}, \gamma_{1}>0, \gamma_{2}>0, b \geqslant 0 ; f(t) \equiv 0$ при $t<0 ; t^{\gamma_{2}} f(t)<$ $<M \exp \left(s_{0} t^{\gamma_{1}}\right) ; M, s_{0}$ - постоянные числа (при $\left.t>0\right) ;{ }_{1} \Phi_{1}^{\tau, \beta}$ - обобщённая конфлюэнтная гипергеометрическая функция [6]:

$$
{ }_{1} \Phi_{1}^{\tau, \beta}(a, c ; z)=\frac{\Gamma(c)}{\Gamma(a) \Gamma(c-a)} \int_{0}^{1} t^{a-1}(1-t)^{c-a-1}{ }_{1} \Psi_{1}\left[\begin{array}{c}
(c, \tau) \\
(c, \beta)
\end{array} \mid z t^{\tau}\right] d t,
$$

$\operatorname{Re} c>\operatorname{Re} a>0, \quad \tau>0, \quad \tau \in \mathbb{R}, \quad \beta>0, \quad \beta \in \mathbb{R}, \quad \tau-\beta<1 ;$

$$
\Gamma(z)=\int_{0}^{\infty} e^{-t} t^{z-1} d t
$$

- классическая Гамма-функция;

$$
{ }_{1} \Psi_{1}\left[\begin{array}{c}
(c, \tau) \\
(c, \beta)
\end{array} \mid z t^{\tau}\right]=\sum_{n=0}^{\infty} \frac{\Gamma(c+\tau n)}{\Gamma(c+\beta n)} \frac{z^{n} t^{\tau n}}{n !}
$$

- частный случай функции Райта [7].

Заметим, что при $b=0$ преобразование (1) совпадает с классическим преобразованием Лапласа [1]:

$$
\mathcal{L}\{f(t) ; x\}=\int_{0}^{\infty} \exp (-t x) f(t) d t .
$$

Аналогично при $\gamma_{2}=0, \gamma_{1}=1, b=0$ преобразование (2) совпадает с $(3)$.

В работах $[8,9]$ приведены и доказаны формулы обращения для интегральных преобразований (1), (2). Например, для интегрального преобразования (1) справедлива формула

$$
f(u)=\frac{\Gamma(a)}{\Gamma(c)} \int_{0}^{\infty}(u x)^{-1} g(x) \mathcal{K}(u x) d x,
$$

где

$$
\begin{gathered}
\mathcal{K}(x)=\frac{1}{2 \pi i} \int_{\sigma-i \infty}^{\sigma+i \infty} \frac{x^{s}}{\xi(s)} d s, \quad g(y)=\widetilde{\mathcal{L}}\{f(x) ; y\}, \\
\xi(s)={ }_{2} \Psi_{1}\left[\begin{array}{c}
(a, \tau) ;(s, \gamma) \\
(c, \beta)
\end{array} \mid-b\right] .
\end{gathered}
$$

Теорема 1. При условиях $\operatorname{Re} c>\operatorname{Re} a>0, \tau \in \mathbb{R}, \tau>0, \beta \in \mathbb{R}, \beta>0$, $\tau-\beta<1, h(x) \in L(0,+\infty)$ интегральное уравнение Вольтерра первого рода

$$
\int_{0}^{x} g(t)(x-t)_{1}^{\gamma-1} \Phi_{1}^{\tau, \beta}\left(a ; c ;-b(s t)^{\gamma}\right) d t=h(x)
$$


имеет единственное решение

$$
g(t)=\frac{\Gamma(a)}{\Gamma(c) \Gamma(\gamma)} \int_{0}^{\infty} x^{\gamma-1} t^{-1} \mathcal{K}(x t) \cdot \mathcal{L}\{h(y) ; x\} d x
$$

Доказ ательств о. Применим к обеим частям уравнения (5) преобразование Лапласа:

$$
\int_{0}^{\infty} e^{-x s}\left(\int_{0}^{x} g(t)(x-t)^{\gamma-1} \Phi_{1}^{\tau, \beta}\left(a ; c ;-b(s t)^{\gamma}\right) d t\right) d x=\mathcal{L}\{h(x) ; s\} .
$$

Непосредственными вычислениями убеждаемся в справедливости равенства

$$
\mathcal{L}\{h(x) ; s\}=\widetilde{\mathcal{L}}\{g(x) ; s\} \cdot \mathcal{L}\left\{x^{\gamma-1} ; s\right\}
$$

Учитывая, что [1]

$$
\mathcal{L}\left\{x^{\gamma-1} ; s\right\}=\frac{\Gamma(\gamma)}{s^{\gamma}}
$$

будем иметь

$$
\widetilde{\mathcal{L}}\{g(t) ; s\}=\frac{s^{\gamma}}{\Gamma(\gamma)} \cdot \mathcal{L}\{h(t) ; s\}
$$

Применяя формулу обращения (4) для интегрального преобразования $\widetilde{\mathcal{L}}\{g(t) ; s\}$, получим искомую функцию $(6)$.

ТЕорема 2. При условиях существования интегралов

$$
\begin{aligned}
& \varphi(s)=\mathcal{L}_{m}\{f(t) ; s\}=\int_{0}^{\infty} t^{m-1} e^{-(t s)^{m}} f(t) d t \\
& \psi(s)=\mathcal{L}_{m}\{g(t) ; s\}=\int_{0}^{\infty} t^{m-1} e^{-(t s)^{m}} g(t) d t
\end{aligned}
$$

и их абсолютной сходимости имеет место следующее равенство:

$$
\mathcal{L}_{m}\{F(x) ; s\}=\mathcal{L}_{m}\{f(t) ; s\} \cdot \mathcal{L}_{m}\{g(t) ; s\}
$$

¿əe

$$
F(x)=\int_{0}^{x} y^{m-1} g(y) f\left(\sqrt[m]{x^{m}-y^{m}}\right) d y
$$

Доказательств о. Здесь $f(t)=0$ и $g(t)=0$ при $t<0$. При доказательстве формулы $(7)$ воспользуемся формулой обращения для интегрального преобразования $\mathcal{L}_{m}$ :

$$
f(t)=\frac{m}{2 \pi i} \int_{\sigma-i \infty}^{\sigma+i \infty} \varphi\left(s^{1 / m}\right) e^{s t^{m}} d s
$$

Рассмотрим интеграл 


$$
\begin{aligned}
\frac{m}{2 \pi i} \int_{\sigma-i \infty}^{\sigma+i \infty} & \varphi\left(s^{1 / m}\right) \psi\left(s^{1 / m}\right) e^{s t^{m}} d s= \\
= & \frac{m}{2 \pi i} \int_{\sigma-i \infty}^{\sigma+i \infty} \varphi\left(s^{1 / m}\right) e^{s t^{m}}\left(\int_{0}^{\infty} y^{m-1} e^{-s y^{m}} g(y) d y\right) d s= \\
= & \int_{0}^{\infty} g(y) y^{m-1} d y\left(\frac{m}{2 \pi i} \int_{\sigma-i \infty}^{\sigma+i \infty} e^{s\left(t^{m}-y^{m}\right)} \varphi\left(s^{1 / m}\right) d s\right)= \\
= & \int_{0}^{\infty} g(y) y^{m-1} f\left(\sqrt[m]{t^{m}-y^{m}}\right) d y=\int_{0}^{t} y^{m-1} g(y) f\left(\sqrt[m]{t^{m}-y^{m}}\right) d y
\end{aligned}
$$

так как $f(t)=0$ при $t<0$.

Введём обозначение

$$
f * g=\int_{0}^{t} y^{m-1} g(y) f\left(\sqrt[m]{t^{m}-y^{m}}\right) d y .
$$

Тогда формула (7) запишется в виде

$$
\mathcal{L}_{m}\{f * g ; s\}=\varphi(s) \cdot \psi(s)
$$

Рассмотрим применение формулы (7) для решения интегрального уравнения, содержащего в ядре обобщённую конфлюэнтную гипергеометрическую функцию:

$$
\int_{0}^{x} y^{m-1}\left(x^{m}-y^{m}\right)^{\gamma / m} \Phi_{1}^{\tau, \beta}\left(a ; c ;-b\left(x^{m}-y^{m}\right)^{\gamma_{1} / m}\right) \cdot g(y) d y=F(x) .
$$

Применим к обеим частям уравнения (8) интегральное преобразование $\mathcal{L}_{m}$.

Поскольку

$$
f\left(\left(x^{m}-y^{m}\right)^{1 / m}\right)=\left(x^{m}-y^{m}\right)^{1 / m} \cdot{ }_{1} \Phi_{1}^{\tau, \beta}\left(a ; c ;-b\left(x^{m}-y^{m}\right)^{1 / m}\right),
$$

в силу равенства (7) получим

$$
\mathcal{L}_{m}\{f(z) ; s\} \cdot \mathcal{L}_{m}\{g(t) ; s\}=\mathcal{L}_{m}\{F(x) ; s\},
$$

где $z=\left(x^{m}-y^{m}\right)^{1 / m}$, то есть $f(z)=z^{\gamma}{ }_{1} \Phi_{1}^{\tau, \beta}\left(a ; c ;-b z^{\gamma_{1}}\right)$.

Вычислим

$$
\begin{aligned}
\mathcal{L}_{m}\left\{z^{\gamma}{ }_{1} \Phi_{1}^{\tau, \beta}\right. & \left.\left(a ; c ;-b z^{\gamma_{1}}\right) ; s\right\}=\int_{0}^{\infty} z^{m-1} e^{-z^{m} s^{m}} z^{\gamma}{ }_{1} \Phi_{1}^{\tau, \beta}\left(a ; c ;-b z^{\gamma_{1}}\right) d z= \\
= & \frac{\Gamma(c)}{\Gamma(a)} \int_{0}^{\infty} z^{m-1+\gamma} e^{-z^{m} s^{m}} \cdot \sum_{n=0}^{\infty} \frac{\Gamma(a+\tau n)}{\Gamma(c+\beta n)} \frac{\left(-b z^{\gamma_{1}}\right)^{n}}{n !} d z= \\
= & \frac{\Gamma(c)}{\Gamma(a)} \sum_{n=0}^{\infty} \frac{\Gamma(a+\tau n)}{\Gamma(c+\beta n)} \frac{(-b)^{n}}{n !} \int_{0}^{\infty} e^{-z^{m} s^{m}} z^{m-1+\gamma+\gamma_{1} n} d z=
\end{aligned}
$$




$$
\begin{gathered}
=\frac{\Gamma(c)}{\Gamma(a)} \frac{1}{m s^{m+\gamma}} \sum_{n=0}^{\infty} \frac{\Gamma(a+\tau n) \Gamma\left(\frac{\gamma+\gamma_{1} n}{m}+1\right)}{\Gamma(c+\beta n)} \cdot \frac{(-b)^{n}}{n ! s^{\gamma_{1} n}}= \\
=\frac{1}{m s^{m+\gamma}} \frac{\Gamma(c)}{\Gamma(a)} \cdot{ }_{2} \Psi_{1}\left[\begin{array}{c}
\left.(a ; \tau) ;\left(\frac{\gamma}{m}+1 ; \frac{\gamma_{1}}{m}\right) \mid \frac{-b}{s^{\gamma_{1}}}\right] \\
(c ; \beta)
\end{array}\right.
\end{gathered}
$$

Здесь использовалась замена переменной $z^{m} s^{m}=t$.

Следовательно, уравнение (9) можно записать в виде

$$
\frac{1}{m s^{m+\gamma}} \frac{\Gamma(c)}{\Gamma(a)}{ }_{2} \Psi_{1}\left[\begin{array}{c|c}
(a ; \tau) ;\left(\frac{\gamma}{m}+1 ; \frac{\gamma_{1}}{m}\right) & \frac{-b}{s^{\gamma_{1}}}
\end{array}\right] \cdot \mathcal{L}_{m}\{g(t) ; s\}=\mathcal{L}_{m}\{F(x) ; s\} .
$$

\section{Отсюда}

$$
\mathcal{L}_{m}\{g(t) ; s\}=m s^{m+\gamma} \frac{\Gamma(a)}{\Gamma(c)}\left({ }_{2} \Psi_{1}\left[\begin{array}{c}
(a ; \tau) ;\left(\frac{\gamma}{m}+1 ; \frac{\gamma_{1}}{m}\right) \\
(c ; \beta)
\end{array} \mid \frac{-b}{s^{\gamma_{1}}}\right]\right)^{-1} \cdot \mathcal{L}_{m}\{F(x) ; s\} .
$$

Применяя формулу обращения для преобразования $\mathcal{L}_{m}$, получим решение уравнения (8):

$$
\begin{aligned}
& g(t)=\frac{m^{2}}{2 \pi i} \frac{\Gamma(a)}{\Gamma(c)} \int_{\sigma-i \infty}^{\sigma+i \infty} e^{s t^{m}}{ }_{s}(m+\gamma) / m\left({ }_{2} \Psi_{1}\left[\begin{array}{c}
(a ; \tau) ;\left(\frac{\gamma}{m}+1 ; \frac{\gamma_{1}}{m}\right) \\
(c ; \beta)
\end{array} \mid \frac{-b}{s^{\frac{\gamma_{1}}{m}}}\right]\right)^{-1} \times \\
& \times \mathcal{L}_{m}\left\{F(x) ; s^{1 / m}\right\} d s .
\end{aligned}
$$

\section{СПИСОК ЛИТЕРАТУРЫ/ REFERENCES}

1. A. Erdélyi, Tables of Integral Transforms (Bateman Manuscript Project), New York, McGraw-Hill, 1954 (vol. 1, Moscow, Nauka, 1969; vol. 2, Moscow, Nauka, 1970 [Russian translation]).

2. В. А. Диткин, А. П. Прудников, Интегральные преобразования и операционное исчисление, М.: Наука, 1974. 542 с.; V. A. Ditkin, A. P. Prudnikov, Integral transforms and operational calculus, International series of monographs in pure and applied mathematics, vol. 78, Oxford, New York, Pergamon Press, 1965, xi+529 pp.

3. A. A. Kilbas, M. Saigo, H-Transforms: Theory and Applications, Series on Analytic Methods and Special Functions, vol. 9, Boca Raton, CRC Press, 2004, xii+389 pp.

4. I. N. Sneddon, The use of integral transforms, New York etc., McGraw-Hill Book Comp., 1972, xii+539 pp.

5. Н. О. Вірченко, Парні (N-арні) інтегральні рівняння, Київ: Задруга, 2009. 476 с. [N. O. Virchenko, Parni ( $N$-arni) integral'ni rivnyannya [Pairs ( $N$-ary) integral equations], Kiev, Zadruga, 2009, 476 pp. (In Ukrainian)]

6. N. Virchenko, "On the generalized confluent hypergeometric function and its applications", Fract. Calc. Appl. Anal., 2006, vol. 9, no. 2, pp. 101-108.

7. E. M. Wright, "The Asymptotic Expansion of the Generalized Hypergeometric Function", J. London Math. Soc., 1935, vol.s1-10, no. 4, pp. 286-293. doi: 10.1112/jlms/s1-10.40.286.

8. О. А. Репин, С. М. Заикина, "Некоторые новые обобщенные интегральные преобразования и их применение в теории дифференциальных уравнений" // Вестн. Сам. гос. техн. ун-та. Сер. Физ.-мат. науки, 2011. №2(23). С. 8-16. doi:10.14498/vsgtu913. [O. A. Repin, S. M. Zaikina, "Some new generalized integral transformations and their application in differential equations theory", Vestn. Samar. Gos. Tekhn. Univ. Ser. Fiz.Mat. Nauki, 2011, no. 2(23), pp. 8-16. (In Russian)]. 
9. N. Virchenko, S. L. Kalla, S. Zaikina, "On some generalized integral transforms", Handronic Journal, 2009, vol. 32, no. 5, pp. 539-548.

Поступила в редакцию 30/IX/2013;

в окончательном варианте - 05/XII/2013;

принята в печать $-17 / \mathrm{I} / 2014$.

MSC: 44A10, 44A20; 33C15, 33C20

\title{
GENERALIZED INTEGRAL LAPLACE TRANSFORM AND ITS APPLICATION TO SOLVING SOME INTEGRAL EQUATIONS
}

\author{
S. M. Zaikina ${ }^{1,2}$ \\ 1 Samara State Technical University, \\ 244, Molodogvardeyskaya st., Samara, 443100, Russian Federation. \\ 2 Volgograd State University, \\ 100, pr-t Universitetskiy, Volgograd, 400062, Russian Federation.
}

We present integral transforms $\widetilde{\mathcal{L}}\{f(t) ; x\}$ and $\widetilde{\mathcal{L}}_{\gamma_{1}, \gamma_{2}, \gamma}\{f(t) ; x\}$, generalizing the classical Laplace transform. The $(\tau, \beta)$ - generalized confluent hypergeometric functions are the kernels of these integral transforms. At certain values of the parameters these transforms coincides with the famous classical Laplace transform. The inverse formula for the transforms is given. The convolution theorem for transform $\widetilde{\mathcal{L}}\{f(t) ; x\}$ is proven. Volterra integral equations of the first kind with core containing the generalized confluent hypergeometric function ${ }_{1} \Phi_{1}^{\tau, \beta}(a ; c ; z)$ are considered. The above equation is solved by the method of integral transforms. The treatment of integral transforms is applied to get the desired solution of the integral equation. The solution is obtained in explicit form.

Keywords: Laplace integral transform, integral equations, generalized hypergeometric function.

\author{
Received 30/IX/2013; \\ received in revised form $05 / \mathrm{XII} / 2013$; \\ accepted $17 / \mathrm{I} / 2014$.
}

ISSN: 2310-7081 (online), 1991-8615 (print); doi: http://dx.doi.org/10.14498/vsgtu1265

(C) 2014 Samara State Technical University.

Citation: S. M. Zaikina, "Generalized Integral Laplace Transform and Its Application to Solving Some Integral Equations", Vestn. Samar. Gos. Tekhn. Univ., Ser. Fiz.-Mat. Nauki [J. Samara State Tech. Univ., Ser. Phys. \& Math. Sci.], 2014, no. 1(34), pp. 19-24. doi: 10.14498/vsgtu1265. (In Russian)

Author Details: Svetlana M. Zaikina, Postgraduate Student, Dept. of Applied Mathematics \& Computer Science ${ }^{1}$; Assistant, Dept. of Computer Science \& and Experimental Mathematics $^{2}$.

E-mail address: svetzai@inbox.ru 